\title{
Effect of Concentration of Ionic Liquids on Resolution of Nucleotides in Reversed-phase Liquid Chromatography
}

\author{
Jin Chun Hua, Yulia Polyakova, and Kyung Ho Row \\ Center for Athanced Bioseparation Technology and Department of Chemical Engineering, \\ Inha Lnwersity, Incheon t02-751, Korea. ${ }^{*}$ E-mail: row kho inha.ack \\ Received January 5, 2007
}

\begin{abstract}
The chromatographic behaviors of nucleotides (inosine 5'-monophosphate. uridine 5'-monophosphate. guanosine 5'-monophosphate and thymine monophosphate disodium salts) on a $\mathrm{C}_{18}$ column were studied with different types of ionic liquids (ILs) as additives for the mobile phase in reversed-phase liquid chromatography (RPLC). Three ILs. 1-butyl-3-methylimidazolium tetrafluoroborate ([BMm] $\left.\left[\mathrm{BF}_{+}\right]\right)$. 1-ethyl-3-methylimidazolium tetrafluoroborate $\left.\left([\mathrm{EMIm}] \mathrm{BF}_{4}\right]\right)$. and 1-ethyl-3-methylimidazolium methylsulfate ([EMIm] [MS]). were used. Eluents were composed of water and methanol $(90 / 10 \%$ vol) with the addition of $0.5-13.0 \mathrm{~m} / \mathrm{of}$ ILs. The effects of the concentration of ILs on retention and separation were investigated and discussed. The results showed that the addition of $L s$ affects the retention and resolution of the tested compounds. Use of 13.0 $\mathrm{m} / \mathrm{f}$ of $[\mathrm{BMIm}]\left[\mathrm{BF}_{4}\right]$ as the eluent modifier resulted in a baseline separation of nucleotides without requiring gradient elution. This study demonstrates that ILs can be potentially applied as a mobile phase modifier in RPLC.
\end{abstract}

Key Words : Ionic liquid. Mobile phase composition. Modifier. Nucleotides, RPLC

\section{Introduction}

Separation science has been hard-pressed to keep pace with the increased sample complexity and analy sis numbers brought about by extensive growth in life sciences initiatives and biomedical advances. Further challenges are being confronted in the envirommental. food and agriculture sectors to address fundamental security and safety issues. As samples of ever-increasing complexity need to be analyzed increasingly large numbers. the need for more universal separation methods and selection of mobile phase becomes paramount. The choice of the organic solvent media requires knowledge of its physical and chemical properties. as well as knowledge of how it impacts the retention of analyte including both interactions with the analytes and the stationary phase.

Methanol is widely used as an organic modifier in reversed-phase liquid chromatography (RPLC). Unfortunately. such simple biphasic systems (water-methanol eluents) do not satisfy the needs of analy sts in the vast majority of cases. The majority of separations are impossible without various mobile phase additives. such as acids. salts. and organic matters. Careful choice of an organic modifier is required in order to fix the elution force of the mobile phase. Variation of the nature and the concentration of the organic modifier affects the interaction energy of the analyte in the bulk mobile phase. In recent years. interest in new modifiers has grown rapidly:

Ionic liquids (ILs) are a type of salt that are liquid at low temperature $(<293 \mathrm{~K})$. In recent years. ILs have been successfully applied in various areas of analytical chemistry. catalysis. " and other applications. ${ }^{3}$ ILs have a variety of desirable properties. such as negligible vapor pressure. good thermal stability. tunable viscosity. and miscibility with water and organic solvents. In addition. it is anticipated that ILs can be applied to sample preparation procedures.

There has been a significant increase in research into other properties of ILs since they were proposed for use as new media for chemical reactions. Building on the advantages of the special properties of ILs. it is expected that the number of mobile phase additives in RPLC can be expanded. Numerous works describing successful applications of ILs in this field are reported annually: ${ }^{4-18}$ Nucleotides in particular are a subject of study in many fields of science. such as biology, chenistry, and medicine and are known to play critical roles in many processes ${ }^{19}$ Many RPLC methods have been applied to the analysis of nucleotides. ${ }^{8.20 .21}$ In previous papers. ${ }^{2-2-24}$ Row et al. investigated the separation of nucleic compounds with different types of mobile phases and modifiers in RPLC. It must be emphasized that in the all previously experiments only common modifiers were used and ionic liquids have never been applied for this purpose. It is reasonably safe to suggest that the present study is a pioneer work and should be of immediate interest to separation science.

Generally, water-organic mobile phases adjusted by modifiers are used in this regard. ${ }^{-5}$ It can reasonably be assumed that most of these modifiers are volatile. and salt out. Here it should be noted that nucleotides are polar compounds and it is clear that their separation requires a stationary phase with low silanol activity and eluents with high ionic strength. It is now well understood that ILs present both major challenges and massive potential for research in this field.

In this study. three ILs were evaluated as mobile phase additives for separation of mixtures composed from four nucleotides by RPLC. The retention factors of solutes were determined with a water/methanol mobile phase comprised 
of ILs on a reversed-phase column without gradient elution. The effects of the nature and concentration of ILs on the chromatographic perfomance are discussed. It appears that the mechanism of separation involves interactions between nucleotides and the ILs.

\section{Experimental Section}

Apparatus. An instrument with an analytical HPLC system was used an M930D solvent delivery module equipped with a M930D solvent delivery pump (Young-In Co. South Korea) a UV M720 absorbance detector (YoungIn Scientific Co. South Korea), and a Reodyne injection (Cotati. CA. USA) valve with a $20 \mu \mathrm{L}$ sample loop. Experiments were perfonmed with a commercially asailable Optimapak $\mathrm{C}_{18}$ (alkyl-) bonded phase colunun $(4.6 \times 250 \mathrm{~mm}$ i.d. and $5 \mathrm{~km}$ particles) from Rs-Tech Co. (Daejeon. South Korea). Chromate software (Ver. 3.0 Interface Eng.. South Korea) on a PC was used as a data acquisition system.

Reagents. Three ILs (99.99\%). 1-butyl-3-methylimidazolium tetrafluoroborate. 1-ethyl-3-methylimidazolium tetrafluoroborate. and 1-ethyl-3-methylimidazolium methylsulfate. were purchased from C-tri Co. (Namyang. South Korea) and are shown in Table 1. Nucleotide standards (uridine 5'monophosphate disodium salt. inosine 5'-monophosphate disodium salt guanosine 5'-monophosphate disodium salt. and thy'midine 5'-monophosphate disodium salt) (Table 2) were purchased from Fluka (St. Louis. MO. USA). All substances were of analytical grade. Potassium nitrate $\left(\mathrm{KNO}_{3}\right)$ was obtained from Kanto Chemical Co. (Japan) and HPLCgradient grade methanol was purchased from Duksan Pure Chemical Co. (Ansan. South Korea). The distilled water was filtered with a vacuum pump (Division of Millipore. Waters. U.S.A.) and a filter (HA-0.45. Division of Millipore. Waters. U.S.A.) prior to use.

Chromatographic conditions. Each analyte was dissolved in water to a concentration of $1000 \mu \mathrm{g} / \mathrm{mLL}$. The misture of nucleotides was prepared as aliquots of individual solutions of 5'-IMP. 5'-TMP. 5'-GMP. and 5'-UMP at a ratio of 1:1:1:1. The mixtures were stored at $277 \mathrm{~K}$. It should be emphasized that the working solutions were re-prepared every 3 days so as to avoid potential errors arising from decomposition. The

Table 1. Names and structures of the ionic liquids

\begin{tabular}{|c|c|c|}
\hline Name (Abbreviation) & Cation & Anion \\
\hline $\begin{array}{l}\text { I-Buty]-3-ethylimidazolium } \\
\text { tetratluoroborate } \\
\left(\left[\mathrm{BM}[\mathrm{m}]\left[\mathrm{BF}_{4}\right]\right)\right.\end{array}$ & & \\
\hline $\begin{array}{l}\text { l-Ethy 1-3-methylinidazoliunn } \\
\text { tetratluoroborate } \\
\text { ([EMIm][BFA]) }\end{array}$ & & \\
\hline $\begin{array}{l}\text { l-Ethy l-3-methyluniduzolium } \\
\text { methylsulfate ([EMIm][MS]) }\end{array}$ & & \\
\hline
\end{tabular}

Table 2. Names and structures of the nucleotides

Name (Abbreviation)
Uridine 5'-monophosphate
disodium salt (5'-UMP)
Inosine 5'-monophosphate
disodium salt (5'-IMP)
disodium salt (5'-GMP)
I'hymidine 5'-monophosphate
disodium salt (5'-lMP)

mobile phase was $10.0 \mathrm{vol} \%$ of methanol in water (pure reversed-phase systems). The modified mobile phases were prepared by dissolving known amounts of ILs in the watermethanol eluent. After each experiment with a specified concentration of ILs. and prior to the experiment with the subsequent concentration of modifier, the column was flushed for at least 3 hours to remove the ILs used at the previous concentration and to fully equilibrate the column. The injection volume of the mixture was $4 \mu \mathrm{L}$. Analyses were performed throughout at ambient temperature $(297 \mathrm{~K})$ at a flow rate of $1.0 \mathrm{~mL} / \mathrm{min}$ flowed in isocratic mode. and the elution profiles were monitored at $\lambda$ of $254 \mathrm{~nm}$.

\section{Results and Discussion}

Generally, in RPLC the retention time will increase as the lipophilicity of the substance is increased and as the percentage of organic modifier in the mobile phase is decreased The nature and concentration of any competing modifier added to the mobile phase will detenmine the retention times and elution order for solute. In this work, the nucleotides were used as model compounds in order to study the contributions of ILs adjustament. All organic matters are polar solutes having different affinity to silanols and to the polar groups. Thus, all the employed test solutes were apt to migrate along the chromatographic column due to the mixed retention mechanism. although quantitative proportions between adsorption and partition depended on quantitative proportions of modifier to water and on the chemical nature of the solute molecules.

The retention factors of these nucleotides were determined with a water/methanol mobile phase comprised of three different kinds of ILs. The retention factor $(k)$ is calculated 
by the following equation:

$$
k=\left(\mathrm{t}_{\mathrm{R}}-\mathrm{t}_{\mathrm{i}}\right) / \mathrm{t}_{\mathrm{i}}
$$

Where $t_{R}$ is the retention time of the sample and $t_{1:}$ is the retention time of the non-retained peak (taken as the first deviation of the baseline following injection of $4 \mu \mathrm{L} \mathrm{KNO}_{3}$ ). The retention factors reported in this study are the averages of at least three determinations. The relative error of a single measurement did not exceed $5 \%$. Evaluation of the results of the chromatographic experiment was carried out using mathematical statistic techniques

Effect of ILs concentration. First nucleotide separation with a pure reversed-phase system ( $10.0 \mathrm{vol} \%$ of methanol in water) was examined. In the subsequent steps the influence of the concentration of three different ILs was tested. Figure 1 shows the dependencies of retention factors $(k)$ on the content of [BMIm] $\left[\mathrm{BF}_{4}\right]$ in the eluent. It is readily apparent that an increase of the $[\mathrm{BMIm}]\left[\mathrm{BF}_{4}\right]$ concentration causes increased retention of the sorbates. Identical trends are obtained for all analytes. Furthermore. this modifier has a major impact on the $k$ parameter values. For example. ad-

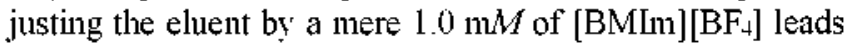
to an increase of $k$ of about two times for all solutes. The analogous tendency was constant until $6.0 \mathrm{mM}$. Increased build-up of the modifier concentration leads to a sharp rise in retention. The $k$ maximums of all solutes with [BMIm] $\left[\mathrm{BF}_{4}\right]$ were obtained at $10.0 \mathrm{mM}$. The greatest $k$ increment is observed for 5'TMP. This is deemed to be a threshold concentration. because the retention of solute decreases slightly with further increase in [BMIm] $\left[\mathrm{BF}_{4}\right]$ concentration. Figure 2 shows chromatograms of nucleotides without (a) and with 0.5 (b). 10.0 (c). and 13.0 (d) $\mathrm{mM}$ of [BMIm] $\left.\mathrm{BF}_{4}\right]$ in $10 \%$ methanol solution. It is clear that three nucleotides (5'-UMP, 5'-IMP, and 5'-GMP) are co-eluted with the unmodified mobile phase (Fig. 2a). It is also noted that the retention times of the solutes are very close to the dead time. The eluent with $13.0 \mathrm{~m} M$ of this modifier provides base-line separation in an acceptable time.

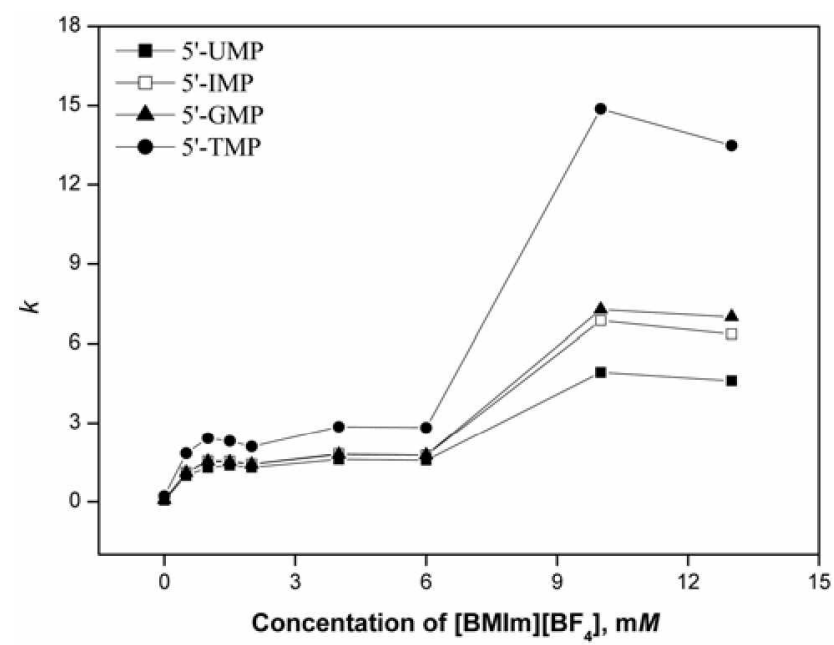

Figure 1. Retention factors $(k)$ of nucleotides without and with [BMIn] $]\left[\mathrm{BF}_{4}\right]$ in mobile phase.

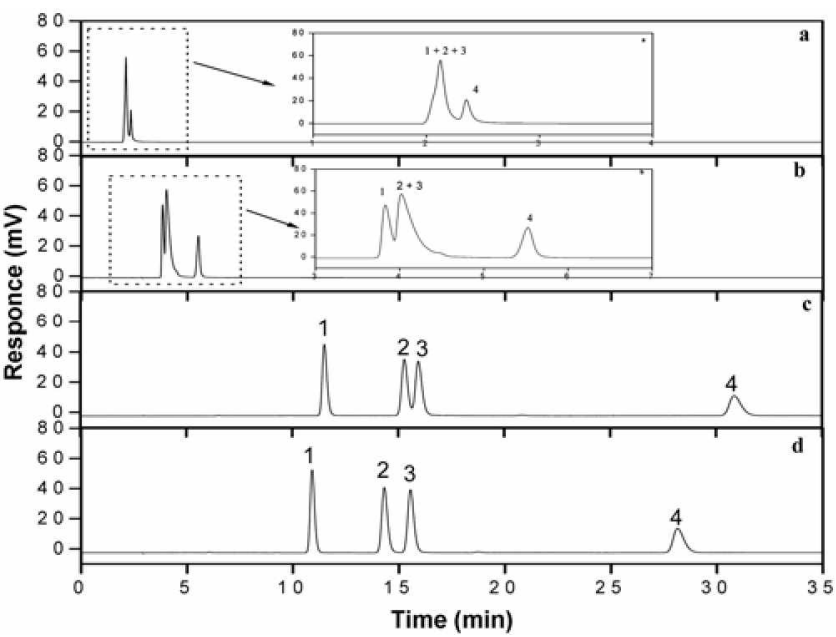

Figure 2. Chromatograms of a mixture of nucleotides with mobile phases without (a) and with 0.5 (b), 10.0 (c) and 13.0 (d) $\mathrm{mM}$ [BMIm] $\left[\mathrm{BF}_{4}\right.$ ] (peaks numbering: (1) 5'-UMP, (2) 5'-IMP, (3) 5'GMP, (4) 5'-TMP).

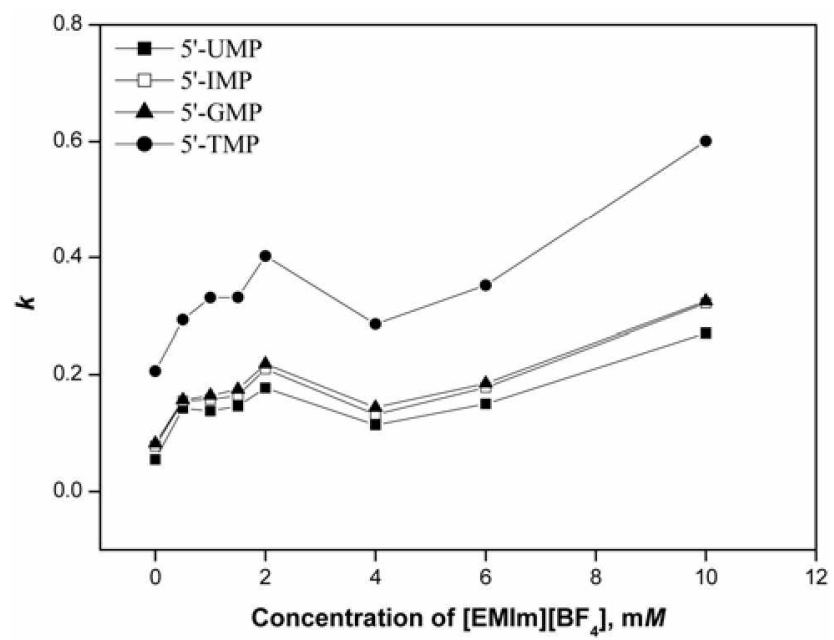

Figure 3. Retention factors $(h)$ of nucleotides without and with $[\mathrm{EMIm}]\left[\mathrm{BF}_{4}\right]$ in mobile phase.

Figure 3 clarifies the effect of [EMIm] $\left[\mathrm{BF}_{4}\right]$ content in the mobile phase on retention. However. the increment of $k$ is much smaller than the earlier modifier $[\mathrm{BMIm}]\left[\mathrm{BF}_{4}\right]$ (Fig. 1). The retention factors with $[\mathrm{EMIm}]\left[\mathrm{BF}_{4}\right]$ are less than 1.0 in all cases. This is not surprising given that the retention times of the solutes were very close to the dead time. The authors believe that some observed maximums and minimums for the trends do not have systematic character. Therefore. the trends appear to be due in part to measurement inaccuracy rather than to physicochemical factors. The analysis was not satisfactory in any case with [EMInı $]\left[\mathrm{BF}_{4}\right]$. as demonstrated by the obtained chromatograms (Fig. 4).

The dependencies of the retention factors $(k)$ on the content of [EMIm][MS] in the eluent are shown in Figure 5. Identical trends are expressed for all analytes with this modifier. In sum, the trend is toward larger retention factors with increased modifier concentration. It is reasonably safe to suggest that the contribution of modifier concentration to 


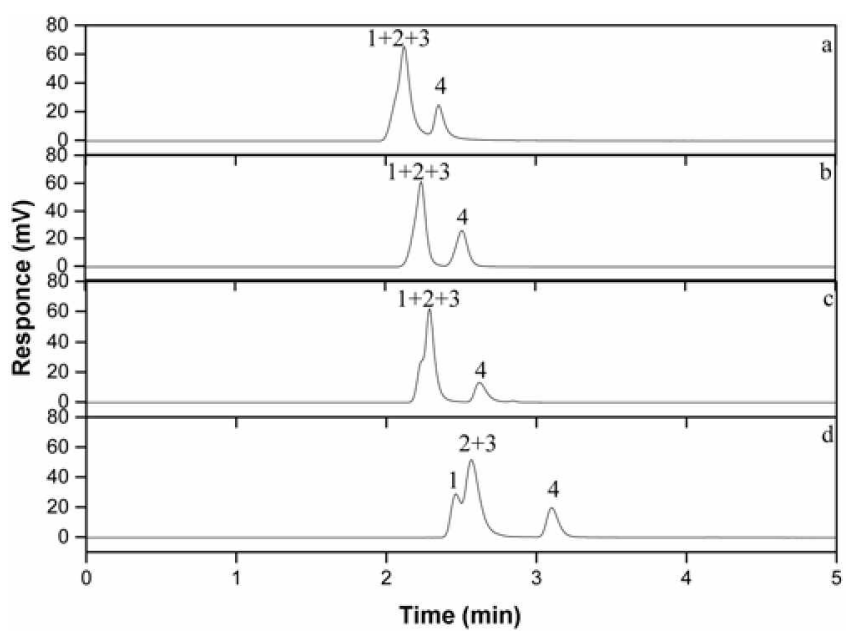

Figure 4 . Chromatograms of a misture of nucleotides with mobile phase without (a) and with 0.5 (b), 60 (c) and 100 (d) $\mathrm{ml}$ [EMIm] $\left[B F_{4}\right]$ (peaks numbering: (1) 5'-UMP, (2) 5'-IMP, (3) 5'GMP, (4) 5'-TMP).

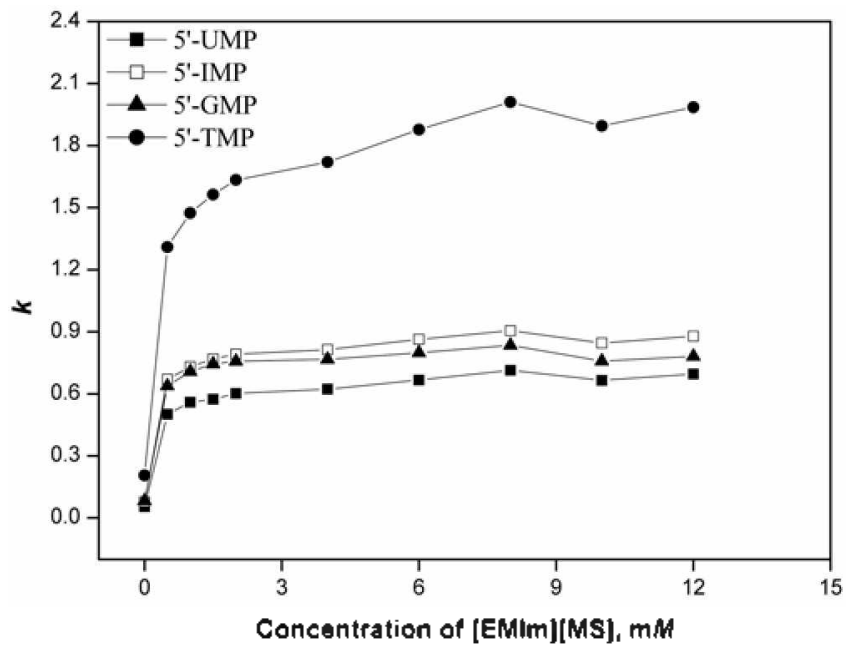

Figure 5. Retention factors $(k)$ of nucleotides without and with $[\mathrm{EM} \mathrm{m}][\mathrm{MS}]$ in mobile phase.

the retention is expressed more at the lowest concentration of [EMIm][MS]. i.e. $0.5 \mathrm{mM}$. In addition, the numerical $k$ values of 5' UMP. 5' IMP, and 5'GMP are very close. In a qualitative sense. for 5 'TMP. we observed the same tendency. In the case of this nucleotide. the retention factor is relatively constant at $6.0 \mathrm{mM}$ of modifier. Chromatograms illustrating isolation without and with [EMIm][MS] in the mobile phase are presented in Figure 6 . From these experiments. it was concluded that the results of nucleotide isolation with IL modifiers are superior to those without modifiers.

Effect of ILs anions. The effects of ILs anions on retention of nucleotides were also evaluated. In this work we tested three ILs. one containing a methylsulfate anion [MS] and two ILs having tetrafluoroborate anions $\left[\mathrm{BF}_{4}\right]$ (Table 1). It is readily seen that two modifiers, $\left.[E M|m|\rfloor B F_{4}\right]$ and [EMlm][MS], have the sanie cations and different anions.

The modifier with [MS] as a counterion provides excellent separation for these nucleotides at the expense of longer

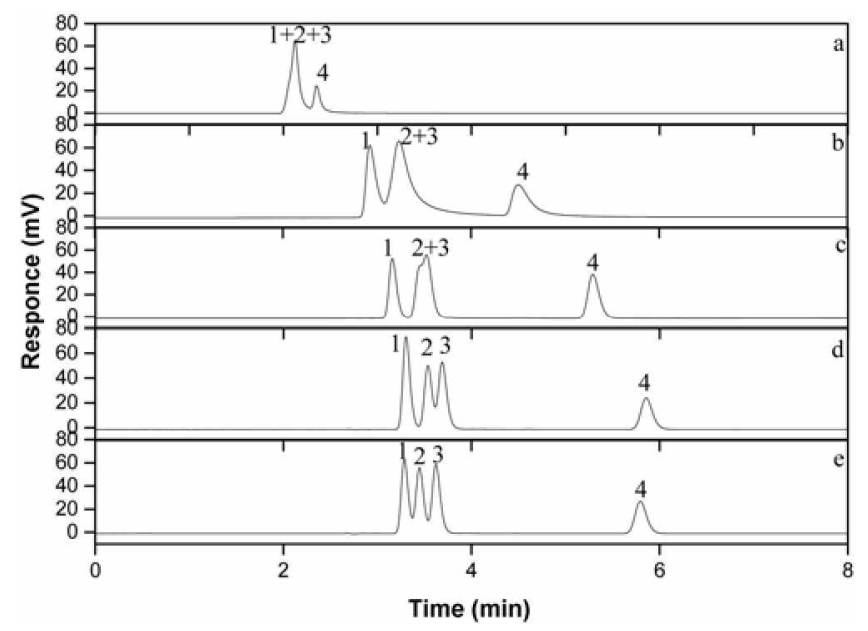

Figure 6. Chromatograms of a mixture of nucleotides with mobile phase without (a) and with 0.5 (b), 4 (c), 8.0 (c) and 12.0 (d) $\mathrm{mm}$ [EMIm][MS] (peaks numbering: (1) 5'-UMP, (2) 5'-IMP, (3) 5'GMP, (4) 5'-TMP)

analysis time in comparison with the modifier combined with $\left[\mathrm{BF}_{4}\right]$. Figures 4 and 6 show chromatograms of solutes on the mobile phase without and with $[E M I m]\left[\mathrm{BF}_{4}\right]$ and [EMIn]][MS], respectively. The solute retention was quite stable and the resolution was not good with the [EMIm]$\left[\mathrm{BF}_{4}\right]$ modifier. The other modifier, [EMIm] [MS]. provides a prepotent effect on the retention. Figures 3 and 5 illustrate the difference dramatically. It is clear that [MS] anion decreases the elution force of the mobile phase. which leads to an increase in the retention time.

ILs are clearly complex entities compared to the relatively simple modifiers used in most chromatographic processes. They are capable of a wider range of intermolecular interactions than most other traditional modifiers of mobile phase. These interactions include: depressiveness. dipolar. inductive. hydrogen bonding. hydrophobic and ionic interactions. A multi-parameter scale that takes into account the many different possible modifier properties can be used to characterize ILs as well as other modifiers.

It is beyond question that the nature of IL anions will have a pronounced effect on the $\mathrm{pH}$ of the eluent media. In a previous paper. ${ }^{26}$ we investigated the $\mathrm{pH}$ of a mobile phase containing different types and concentrations of ILs. It was shown that the $\mathrm{pH}$ of the mobile phase containing $\left[\mathrm{BF}_{4}\right]$ anions was slightly increased with increasing modifier concentration. Conversely, the $\mathrm{pH}$ with [MS] anions decreased with increasing concentration of ILs. The methylsulfate anion includes a hydroxyl group. Thus. this anion can donate hydrogen cations easily, or in other words. invests acidity. However. tetrafluoroborate contains weak basic anions. Accordingly. the acidic working environments formed with [MS] render improved scope for better separation of ionizable solutes. Thereby, the observed differences in retention with $\left[\mathrm{BF}_{4}\right]$ and $[\mathrm{MS}]$ anion-containing modifiers can be explained by changes in the $\mathrm{pH}$ of the eluent media. The different separations that resulted from two ILs with different counterions as modifiers may be due to the association with 
solutes in the eluent. It is illustrated that IL different anions play an important role in separating the nucleotides.

Effect of alkyl groups of the imidazolium cation. In order to provide a satisfactory explanation for the retention. the effects of alkyl groups of the imidazolium cation are discussed. We examined two ILs with l-ethyl-3-methylimidazolium cations and only one modifier contained l-butyl-3methylimidazolium cation (Table 1). Thus. two modifiers. $[\mathrm{BMIm}]\left[\mathrm{BF}_{4}\right]$ and $[\mathrm{EMLm}]\left[\mathrm{BF}_{4}\right]$. have the same anion and different cations.

The modifier with [BMIm] cation (Fig. 2) provides the solution when compared with [EMIm] (Fig. 4). From the preceding results. it is seen that the retention factors with [EMIm] cationic modifiers are less than 1.0 (except for 5'TMP with [EMLm][MS]). Simultaneously, the solutes are most retained with [BMIm]-containing modifier. This solute behavior indicates that the nature of the cation. in particular the length of the hydrocarbonic chain. has a strong influence on the processes of sorbate distribution between stationary and mobile phases. With the presence of [EMIm] cationic modifiers. the interactions of the "solute-eluent" become dominant resulting in low numerical values of $k$. Monomethylation of the cation of the modifier has a significant impact on the energy of the solute interactions with the stationary phase.

Effect of ILs on separation mechanism. According to references. ${ }^{3.14}$ certain molecular interactions. including strong hydrogen bonding (the hydrogen on $\mathrm{C}-2$ carbon of the imidazolium cation), hydrophobic interactions and iondipole or ion-induced-dipole. strong ion-pairing effects. etc. were found in ILs. Accordingly, as mobile phase additives. ILs can play a variety of roles. including coating residual silanols. modification of the stationary phase. and acting as ion-pairing agents. According to $\mathrm{He}$ et al. ${ }^{5}$ ILs form a layer (pseudo-stationary phase) on the surface of the modified silica gel. Previous studies s. $^{-9.11 .13}$ have shown that IL liquid cations can interact and compete with silanol groups on an alkyl silica base surface. The absorption of IL cations by the lipophilic part of alkyl chains of the sorbent is even more likely: Modification by ILs creates an electrostatic potential on the surface. thus providing an additional retentive force for ionized analytes. It is possible that the surface acquires some ion-exchange properties. and retention is subordinated to laws governing ion exchange chromatography. The degree of retention of the analyte decreases with an increase in the ionic force of the mobile phase and increases with an increase in the ion-exchange capacity' of the surface. Thus. the Il modifier disables the alkyl groups of the stationary phase. which leads to a sharp decrease in the possibility of dispersion interactions between the sorbate and the alkyl groups of the stationary phase

Hence. the retention time of nucleotides changes due to certain molecular interactions between nucleotides and ILs. The degree of retention of the nucleotides decreases with an increase in the ionic force of the mobile phase and increases with an increase in the capacity of the surface. The visually observable improvements in the peak tailing (Figs. 2. 4. and
6) indicate that part of the cations preferentially adsorb on the $C_{18}$ stationary phase by covering active silanol sites, which leads to improvement of the peak characteristics. The constitutive anion of a given IL may have a very different effect compared to the associated cation. The inclusive effect of the two oppositely charged ions may be synergistic or antagonist. Thus. a sorbate. which exists in the solution as an anion. is capable of specific ionic interactions with the sorbed cations of ILs. This chromatographic behavior can be explained by the realization of the ion-exchange mechanism of retention.

From the results presented here it is evident that the effect of IL modifiers on separation can be very complex. It is probable that there is not a single solute retention mechanism in chromatography and certainly pure mechanisms with modified eluents cannot be expected. It would. however. be difficult to find the underlying mechanism concerning the nature of modifiers. for which adsorption-partition becomes a multiplex mechanism. It is not inconceivable that new separation mechanisms can be made available with ILs. These aspects can enable the development of more efficient separation processes. The use of ILs should also increase the number of water-miscible modifiers that can be used for RPLC. The influence of ILs on the separation mechanism in RPLC is currently under investigation and discussion. The successful examples of application of ILs to chromatographic separations provide a powerful stimulus for further studies in this direction.

\section{Conclusion}

In this study. successful separation of four nucleotides has been achieved using 1-alkyl-3-methylimidazolium-based ILs as mobile phase modifiers. ILs showed promising performance as additives in RPLC. Excellent separation of nucleotides was achieved using $13.0 \mathrm{~m} M$ [BMIm] $\left[\mathrm{BF}_{4}\right]$ as the eluent modifier. From the results. it is apparent that application of ILs to RPLC provides an effective means of separating ionizable solutes. In order to draw more general conclusions regarding the effect of such modifiers on the retention mechanisms in RPLC, more data are required. particularly results with other ILs modifiers.

Acknowledgement. This work was supported by INHA UNIVERSITY Research Grant (INHA-2007).

\section{References}

1. Liu. T.: Tonssont. T.: Tiang. G. TrAC Thend Anal Chem, 2005. 24. 20.

2. Zhao. D.: Wu. M.: Kou, Y: Min, E. Catal Today 2002. 74. 157

3. Huddleston, J. G.: Visser. A. E.; Reichert. W. M.: Willauer. H. D.; Broker. G. A.; Rogers. R. D. Green Chem. 2001, t, 156.

4. Poole. C. F; Kersten, B. R.: Ho. S. S. J.: Coddens, M. E:; Furton. K. G. J. Chomatogr. A 1986. 352.407.

5. He. L.: Zhang. W.: Zhao. L.: Liu. X.: Tiang. S. J. Chronatogr A 2003. 1007.39 .

6. Zhang. W.: He. L.; Gu, Y.: Liu, X:; Jiang. S. Anal Lett. 2003. 36. 827. 
7. Xiao. X. H.: Liang. Z.: Liu. X: Jiang. S. X. Anot. Chim .Acta 2004. 519.207

8. Zhang. W.: He. L.: Liu. X.: Jiang. S. Chin. J. Chemi. 2004. 22. 549.

9. Marszall, M. P. Biczek, T.: Kaliszan. R. Anat Chim Acta 2005. 547,172 .

10. Polvakota. Y.: Koo, Y. M: Row K. H. Biotechmol Bioprocess Eng. 2006. H. 1.

11. Polyakova. Y.: Jin. Y: Zheng. J.: Row. K. H. J. Liq. Chromatogr: Related Technol. 2006. 29. 1687.

12. Jin, Y.: Lee, T. H.: Polyakota, Y: Koo. Y. M.: Row, K. H. Norea Chem. Eng. Res. 2006. H, 453.

13. Ruiz-Angel. M. I.: Carda-Broch, S.; Berthod, A. J. Chromatogr: A 2006. 1119.202

14. Yanes. E. G.: Gratz. S. R.: Baldwin. M. J.: Robison. S. E.: Stalcup. A. M. Anat Chem. 2001. 73. 3838.

15. Imaz. C.: Carreras, D.: Navajas. R: Rodriguez, C.: Rodriguez, A. F: Maynar. I.: Cortes. R. J. Chromatogr . 1 1993, 631, 201.

16. Imaz. C.: Navajas. R: Carreras, D.: Rodriguez, C.J. Chromatogr. A 2000. 870.23 .
17. Stalcup, A. M:- Cabovstia, B. J. Liq. Chomatog Related Techol. 2004. 27. 1443 .

18. Polyakova. Y.: Row. K. H. Acta Chomatogr: 2006. 17.210.

19. Harmon1. R. E.: Townsend. L. B.: Robins. R. K. Chentistry and Biology of Nucleosides and Kucleotides' Academic Press: New: York. 1978: p 468.

20. Brown. P. R. Hplc in Kucleic Acid Research fChromatographic Sciencel: Academic Press: New York. 1984: p 424.

21. Fallon. A.: Booth. R. F. G.: Bell. L. D. Applications of HPLC in Biochentisty: Elsevier: 1987: p 338

22. Zhene. J.: Polvakova, Y.: Row. K. H. Chomatographia 2006. 64. 129 .

23. Jin. L. M.: Choi. D. Y: Wang. Y. N.: Ahn1. W. S.: Choi. D. K.: Row. K. H. J. Ind. Eng. Chem. 2005. H. 4.

24. Kang. D. D.: Lee. I. W.: Row. K. H. J. Liq. Chomatogr Related Technol. 2001. 24.6.

25. Wulfson. A. N.; Yakimov. S. A. J. High Resolut. Chromatogr. 2005. 7,442.

26. Zheng. J. Z.: Polvakova. Y.: Row, K. H. J. Chomatogr: Sci. 2006. in press. 